\title{
Numerical Calculation of Integrals with Strongly Oscillating Integrand
}

\author{
By A. I. van de Vooren and H. J. van Linde
}

Abstract. In this paper a method is presented for evaluating

$$
\int_{0}^{N} f(x) e^{i \omega x} d x \text { where } \omega N=p \cdot 2 \pi, \quad p \text { integer. }
$$

The idea is to approximate $f(x)$ instead of the whole integrand by aid of polynomials. The Romberg-Stiefel algorithm has been extended to this case. The new method is complementary to the usual Romberg-Stiefel algorithm in the sense that it is more advantageous for larger values of $\omega$. An expression for the remainder term is also included. Results for the real part are exact if $f(x)$ is of at most 7th degree and for the imaginary part if $f(x)$ is of at most 8th degree.

1. Introduction. The conventional methods of numerical integration are generally less suitable for the computation of integrals of the form

$$
\int_{0}^{\infty} f(x) \cos \omega x d x \text { and } \int_{0}^{\infty} f(x) \sin \omega x d x,
$$

if $\omega$ is large. Due to the oscillatory character of the integrand, its approximation by aid of polynomials requires a large number of points where the integrand must be evaluated.

In the present paper a method is given where instead of the whole integrand only the function $f(x)$ is approximated by polynomials. Moreover, the purely numerical part of the method is confined to the evaluation of

$$
\int_{0}^{N} f(x) \cos \omega x d x \text { and } \int_{0}^{N} f(x) \sin \omega x d x
$$

where

$$
\omega N=p \cdot 2 \pi \text { and } p \text { an integer. }
$$

The value of $p$ should be chosen so large that the remaining part of the original integrals referring to the interval $(N, \infty)$ can be calculated by asymptotic expansion of $f(x)$.

The idea of the method is to extend Stiefel's method of numerical integration [1] to integrals with strongly oscillating integrands. Stiefel's method can be seen as an iteration method in which the number of points where the integrand must be evaluated, is doubled at each step. As soon as the change in the result is less than a certain tolerance, the process is stopped. Hence, one obtains an automatic check of the accuracy obtained.

In order to retain this principle for the evaluation of the integrals (1.1), where only $f(x)$ is approximated by polynomials, it is necessary that the points where $f(x)$

Received August 30, 1965. 
must be evaluated have fixed positions with regard to the wave occurring in the goniometric function. This leads to the condition (1.2) for the upper integration limit $N$. The maximum number of evaluation points for which in this report formulae for the values of the integrals are presented, is 16 per wavelength. It may be, however, that $f(x)$ is so smooth or $\omega$ so large, that this number is unnecessarily large for the accuracy required. Therefore, it is preferable if $p$ is not only an integer, but if it is some power of 2 . This enables one to perform more iteration steps with the possibility that the process needs not to be continued until the finest division of the interval (16 points per wavelength).

The method works better for larger values of $\omega$ and, in this respect, can be seen as a useful complement to the normal Stiefel routine.

In many cases it may be advisable, as with Stiefel's method, to divide beforehand the total interval from 0 to $N$ in a few parts over which the integrals are calculated separately. This gives the advantage that if somewhere in the interval $f(x)$ is relatively difficult to approximate by polynomials, which then compels to a large number of evaluation points, this same number does not need to be retained everywhere in the interval from 0 to $N$.

Like in Stiefel's method, it is also possible in the present method to derive from two consecutive steps by linear combination a new approximation which is still exact for a polynomial of higher degree (deferred approach to the limit, see e.g. Fox [2]). However, the coefficients occurring in the linear combination are more difficult to calculate in the present method and, moreover, they become dependent on the number of the step. In this paper coefficients are given which lead to exact results if in the cosine-integral $f(x)$ is of at most 7 th degree and in the sine-integral of at most 8 th degree.

For the case of the most accurate formulae presented in this report ( 16 evaluation points per wavelength), the truncation error has been calculated.

The oldest method for the numerical calculation of integrals with oscillating integrands is probably due to Filon [3]. He approximates the function $f(x)$ in parts of the interval by parabola and then calculates the integral with arbitrary limits. This method has been extended by Luke [4], who approximates the function $f(x)$ in a certain interval by a polynomial of at most 10th degree. This makes his method related to the Newton-Cotes integration formulae. The moments

$$
M_{m}=\int x^{m} e^{i \omega x} d x
$$

are calculated by aid of the function

$$
g(\vartheta)=\frac{\sin \vartheta}{\vartheta}
$$

This function as well as its derivatives are in Luke's method available in the form of a table which make it less suitable for a computer. Also, there is no indication of the accuracy of the numerical result obtained. It has, however, the advantage that the points where $f(x)$ must be evaluated are independent of $\omega$. This is not so in the method presented in this report, since here these points have fixed positions with regard to the wavelength.

Hurwitz and Zweifel [5], [6] suggested the application of Gauss-Jacobi quadra- 
ture to obtain numerical approximations of Fourier transforms. Recently, however, it was shown by Sänger [7], that this method is nothing else than the application of the trapezoidal rule to the complete integrand.

2. Integration Formulae for $\int_{0}^{N} f(x) \cos \omega x d x$. Approximation of $f(x)$ by a constant or a linear function yields zero, since the integration is over an integer number of wavelengths. Therefore the first sensible approximation of $f(x)$ is by a parabola, which agrees with $f(x)$ in the points $x=0, N / 2$ and $N$. This leads to the following result for the integral

$$
\mathbf{A}_{1}: \frac{4}{\omega^{2} N}\left\{f(0)-2 f\left(\frac{N}{2}\right)+f(N)\right\} .
$$

Assuming now that the number $p$ of wavelengths in not only an integer, but is equal to a power of two, i.e.

$$
\omega N=2^{n} \pi, \quad n=1,2, \cdots .
$$

we may write the result also in the form

$$
\mathbf{A}_{1}: \frac{2^{2-n}}{\pi \omega}\left\{f(0)-2 f\left(2^{n-1} \frac{\pi}{\omega}\right)+f\left(2^{n} \frac{\pi}{\omega}\right)\right\} .
$$

We can improve this result by doubling the number of points where $f(x)$ has to be evaluated. We then approximate by aid of two parabola, one through the values of $f(x)$ at $x=0, N / 4, N / 2$ and the other through $f(x)$ at $x=N / 2,3 N / 4$ and $N$. This yields

$$
\mathbf{A}_{2}: \frac{2^{3-n}}{\pi \omega}\left\{f(0)-2 f\left(2^{n-2} \frac{\pi}{\omega}\right)+2 f\left(2^{n-1} \frac{\pi}{\omega}\right)-2 f\left(3 \cdot 2^{n-2} \frac{\pi}{\omega}\right)+f\left(2^{n} \frac{\pi}{\omega}\right)\right\} .
$$

We can proceed in the same way, obtaining as next approximation

$$
\begin{aligned}
\mathrm{A}_{3}: \frac{2^{4-n}}{\pi \omega}\left\{f(0)-2 f\left(2^{n-3} \frac{\pi}{\omega}\right)\right. & +2 f\left(2 \cdot 2^{n-3} \frac{\pi}{\omega}\right)-2 f\left(3 \cdot 2^{n-3} \frac{\pi}{\omega}\right) \\
+2 f\left(4 \cdot 2^{n-3} \frac{\pi}{\omega}\right) & -2 f\left(5 \cdot 2^{n-3} \frac{\pi}{\omega}\right)+2 f\left(6 \cdot 2^{n-3} \frac{\pi}{\omega}\right) \\
& \left.-2 f\left(7 \cdot 2^{n-3} \frac{\pi}{\omega}\right)+f\left(8 \cdot 2^{n-3} \frac{\pi}{\omega}\right)\right\} .
\end{aligned}
$$

This can be continued until the $n$th approximation. The $j$ th approximation $(j \leqq n)$ can be written as

$$
\begin{aligned}
\mathrm{A}_{j}: \frac{2^{j+1-n}}{\pi \omega} \sum_{k=1}^{2 j-1}\left[f \left\{(2 k-2) 2^{n-j}\right.\right. & \left.\frac{\pi}{\omega}\right\} \\
& \left.-2 f\left\{(2 k-1) 2^{n-j} \frac{\pi}{\omega}\right\}+f\left\{2 k \cdot 2^{n-j} \frac{\pi}{\omega}\right\}\right] .
\end{aligned}
$$

The last ( $n$ th) approximation of this series becomes

$$
\mathrm{A}_{n}: \frac{2}{\pi \omega} \sum_{k=1}^{p}\left[f\left\{(2 k-2) \frac{\pi}{\omega}\right\}-2 f\left\{(2 k-1) \frac{\pi}{\omega}\right\}+f\left\{2 k \frac{\pi}{\omega}\right\}\right],
$$


where $p$ is not necessarily $2^{n-1}$. In formula (2.6) the function $f(x)$ is approximated by different parabola over each wavelength $2 \pi / \omega$. Hence, the same formula can be retained for any integer value of $p$, which is in agreement with Eq. (1.2).

The next step would be to approximate $f(x)$ over each half wavelength $\pi / \omega$ by a parabola. After evaluation this appears to lead to exactly the same result as the previous approximation and formula (2.6) is again obtained.

Next, the approximation of $f(x)$ by parabola is performed over a quarter of a wavelength. This leads to

$$
\begin{aligned}
\mathrm{A}_{n+1}: \frac{2}{\pi \omega}\left(3-\frac{8}{\pi}\right) \sum_{k=1}^{p}\left[f\left\{(2 k-2) \frac{\pi}{\omega}\right\}-2 f\left\{(2 k-1) \frac{\pi}{\omega}\right\}+f\left\{2 k \frac{\pi}{\omega}\right\}\right] \\
+\frac{8}{\pi \omega}\left(\frac{4}{\pi}-1\right) \sum_{k=1}^{p}\left[f\left\{\left(2 k-\frac{7}{4}\right) \frac{\pi}{\omega}\right\}-f\left\{\left(2 k-\frac{5}{4}\right) \frac{\pi}{\omega}\right\}\right. \\
\left.\quad-f\left\{\left(2 k-\frac{3}{4}\right) \frac{\pi}{\omega}\right\}+f\left\{\left(2 k-\frac{1}{4}\right) \frac{\pi}{\omega}\right\}\right] .
\end{aligned}
$$

Finally, $f(x)$ is approximated over each eighth wavelength by another parabola and we have

$$
\begin{aligned}
& \mathrm{A}_{n+2}: \frac{2}{\pi \omega}(6+\left.\sqrt{2}-\frac{16 \sqrt{2}}{\pi}\right) \sum_{k=1}^{p}\left[f\left\{(2 k-2) \frac{\pi}{\omega}\right\}-2 f\left\{(2 k-1) \frac{\pi}{\omega}\right\}\right. \\
&\left.+f\left\{2 k \frac{\pi}{\omega}\right\}\right]+\frac{4}{\pi \omega}\left(1+3 \sqrt{2}-\frac{16}{\pi}\right) \sum_{k=1}^{p}\left[f\left\{\left(2 k-\frac{7}{4}\right) \frac{\pi}{\omega}\right\}\right. \\
&\left.-f\left\{\left(2 k-\frac{5}{4}\right) \frac{\pi}{\omega}\right\}-f\left\{\left(2 k-\frac{3}{4}\right) \frac{\pi}{\omega}\right\}+f\left\{\left(2 k-\frac{1}{4}\right) \frac{\pi}{\omega}\right\}\right] \\
&+\frac{8}{\pi \omega}\left(\frac{8 \sqrt{2}}{\pi}-2-\sqrt{2}\right) \sum_{k=1}^{p}\left[f\left\{\left(2 k-\frac{15}{8}\right) \frac{\pi}{\omega}\right\}-f\left\{\left(2 k-\frac{9}{8}\right) \frac{\pi}{\omega}\right\}\right. \\
&\left.-f\left\{\left(2 k-\frac{7}{8}\right) \frac{\pi}{\omega}\right\}+f\left\{\left(2 k-\frac{1}{8}\right) \frac{\pi}{\omega}\right\}\right] \\
&+\frac{8}{\pi \omega}\left(\frac{16-8 \sqrt{2}}{\pi}-\sqrt{2}\right) \sum_{k=1}^{p}\left[f\left\{\left(2 k-\frac{13}{8}\right) \frac{\pi}{\omega}\right\}-f\left\{\left(2 k-\frac{11}{8}\right) \frac{\pi}{\omega}\right\}\right. \\
&\left.-f\left\{\left(2 k-\frac{5}{8}\right) \frac{\pi}{\omega}\right\}+f\left\{\left(2 k-\frac{3}{8}\right) \frac{\pi}{\omega}\right\}\right] .
\end{aligned}
$$

The formulae (2.6), (2.7) and (2.8) have the favourable property that for any value of $x$ the contribution of $f(x)$ has the same sign as $f(x) \cos \omega x$. These same formulae can also be used for the evaluation of arbitrary Fourier cosine coefficients of $f(x)$ in the interval $(0, N)$.

Finally, it can easily be shown that all formulae given hitherto are not only exact when $f(x)$ is a parabola but also if it is a third degree polynomial over each wavelength.

Similar to Stiefel's method, it is now possible to obtain integration formulae which are exact for polynomials up to the 5th degree by linear combination of two consecutive results in the $A_{j}$-sequence. Hence, we shall show that coefficients $\alpha_{j}$ and $\alpha_{j}^{\prime}$ can be determined such that

$$
B_{j}=\alpha_{j} A_{j}+\alpha_{j}^{\prime} A_{j+1}
$$


is an approximation for

$$
\int_{0}^{N} f(x) \cos \omega x d x
$$

which is exact if $f(x)$ is a polynomial of at most 5 th degree over intervals of $2^{n-j}$ wavelengths. ${ }^{*}$ First, we shall determine $\alpha_{j}$ and $\alpha_{j}{ }^{\prime}$ such that $B_{j}$ is exact for 4 th degree polynomials $f(x)$. Next, the coefficients $\alpha_{j}$ and $\alpha_{j}^{\prime}$ are uniquely determined by the further requirement that

$$
\alpha_{j}+\alpha_{j}^{\prime}=1
$$

expressing that $B_{j}$ is also exact for 2 nd degree polynomials.

By aid of an argument similar to that which was used to show that the $A_{j}$ results, derived for 2 nd degree polynomials, hold also for 3rd degree polynomials, it follows that the $B_{j}$-results then are also exact for 5 th degree polynomials.

The exact result for $f(x)=x^{4}$ is given by

$$
\int_{0}^{N} x^{4} \cos \omega x d x=\frac{4 N^{3}}{\omega^{2}}-\frac{24 N}{\omega^{4}} .
$$

The approximate result for this same case as following from Eq. (2.5) is

$$
A_{j}: \frac{N^{3}}{\omega^{2}}\left\{4-\frac{2}{2^{j}}\right\}, \quad j=1,2, \cdots,
$$

where use has been made of Eq. (2.1) and of the series

$$
\sum_{k=1}^{m} k^{0}=m, \quad \sum_{k=1}^{m} k=\frac{1}{2} m^{2}+\frac{1}{2} m, \quad \sum_{k=1}^{m} k^{2}=\frac{1}{3} m^{3}+\frac{1}{2} m^{2}+\frac{1}{6} m .
$$

The approximate results for $f(x)=x^{4}$, following from Eqs. (2.7) and (2.8) are

$$
\begin{aligned}
& A_{n+1}: \frac{4 N^{3}}{\omega^{2}}-\frac{N}{\omega^{4}}\left(\frac{\pi^{2}}{2}+6 \pi\right), \\
& A_{n+2}: \frac{4 N^{3}}{\omega^{2}}-\frac{N}{\omega^{4}}\left\{\frac{\pi^{2}}{8}+3 \pi(1+\sqrt{2})\right\},
\end{aligned}
$$

where Eq. (1.2) has been used as well as Eq. (2.11). The coefficients of $N / \omega^{4}$ appearing in the last two formulae are approximately -23.77 and -23.93 , respectively.

The equations (2.9) and (2.10) now form two equations for the unknowns $\alpha_{j}$ and $\alpha_{j}^{\prime}$. For $B_{j}$ the exact result (2.11) is substituted, while $A_{j}$ is given by (2.12), (2.14) and (2.15). We obtain

$$
\begin{aligned}
\alpha_{j} & =-\frac{1}{3}+\frac{16}{\pi^{2}} \frac{2^{2 j}}{2^{2 n}}, \quad \alpha_{j}{ }^{\prime}=\frac{4}{3}-\frac{16}{\pi^{2}} \frac{2^{2 j}}{2^{2 n}}, \quad j=1,2, \cdots, n-1, \\
\alpha_{n} & =-\frac{1}{3}+\frac{16(\pi-3)}{3 \pi(4-\pi)}, \quad \alpha_{n}{ }^{\prime}=\frac{4}{3}-\frac{16(\pi-3)}{3 \pi(4-\pi)}, \\
\alpha_{n+1} & =-\frac{1}{3}+\frac{16(\pi+2 \pi \sqrt{2}-12)}{3 \pi(8 \sqrt{2}-\pi-8)}, \quad \alpha_{n+1}^{\prime}=\frac{4}{3}-\frac{16(\pi+2 \pi \sqrt{2}-12)}{3 \pi(8 \sqrt{2}-\pi-8)} .
\end{aligned}
$$

As mentioned already above, the results obtained in this way are also exact if

* With the exception that for $j=n+1, f(\mathrm{x})$ may be a different 5 th degree polynomial over each quarter wavelength. 
$f(x)$ is a polynomial of 5 th degree. If $f(x)$ is different, the sequence $B_{1}, B_{2}, \cdots, B_{n+1}$ gives results of increasing accuracy.

We can continue this procedure and form a linear combination of $B_{j}$ and $B_{j+1}$ which we make exact for $f(x)$ being a 6th degree polynomial and which then will be exact also for 7 th degree polynomials (over intervals of $2^{n-j}$ wavelengths). This combination is

$$
C_{j}=\beta_{j} B_{j}+\beta_{j}^{\prime} B_{j+1} \text { with } \beta_{j}+\dot{\beta}_{j}^{\prime}=1 .
$$

The exact result for $f(x)=x^{6}$ is

$$
\int_{0}^{N} x^{6} \cos \omega x d x=\frac{6 N^{5}}{\omega^{2}}-\frac{120 N^{3}}{\omega^{4}}+\frac{720 N}{\omega^{6}},
$$

while the approximate results following from Eqs. (2.5), (2.7) and (2.8) are for this case

$$
\begin{aligned}
A_{j}: & \frac{N^{5}}{\omega^{2}}\left(6-\frac{10}{2^{2 j}}+\frac{6}{2^{4 j}}\right), \quad j=1,2, \cdots, n \\
A_{n+1}: & \frac{6 N^{5}}{\omega^{2}}-\left(\frac{5}{2}+\frac{30}{\pi}\right) \pi^{2} \frac{N^{3}}{\omega^{4}}+\left(\frac{69}{64}+\frac{315}{16 \pi}\right) \pi^{4} \frac{N}{\omega^{6}}, \\
A_{n+2}: \frac{6 N^{5}}{\omega^{2}}- & \left(\frac{5}{8}+\frac{15}{\pi}+\frac{15 \sqrt{2}}{\pi}\right) \pi^{2} \frac{N^{3}}{\omega^{4}} \\
& +\left(\frac{159}{1024}+\frac{45 \sqrt{2}}{512}+\frac{1395}{128 \pi}+\frac{1035 \sqrt{2}}{128 \pi}\right) \pi^{4} \frac{N}{\omega^{6}} .
\end{aligned}
$$

The approximate numerical values of the coefficients of $N^{3} / \omega^{4}$ are -118.9 and -119.9 and those of $N / \omega^{6}$ are 716.3 and 720.2 .

Besides Eqs. (2.13) the following series have been used

$$
\sum_{k=1}^{m} k^{3}=\frac{1}{4} m^{4}+\frac{1}{2} m^{8}+\frac{1}{4} m^{2}, \quad \sum_{k=1}^{m} k^{4}=\frac{1}{5} m^{5}+\frac{1}{2} m^{4}+\frac{1}{3} m^{3}-\frac{1}{30} m .
$$

Forming now the $B_{j}$-approximations from (2.9) and (2.16) we obtain

$$
\begin{aligned}
B_{\jmath} & :\left(6-\frac{3}{2^{4 j+1}}\right) \frac{N^{5}}{\omega^{2}}-\left(120-\frac{90}{2^{2 j}}\right) \frac{N^{3}}{\omega^{4}}, \quad j=1,2, \cdots, n-1 \\
B_{n}: & \frac{6 N^{5}}{\omega^{2}}-\frac{120 N^{3}}{\omega^{4}}+\left(-\frac{9}{16} \pi^{4}+\frac{315}{4} \pi^{2}\right) \frac{N}{\omega^{6}}, \\
B_{n+1}: & \frac{6 N^{5}}{\omega^{2}}-\frac{120 N^{3}}{\omega^{4}} \\
+ & \left(-\frac{39}{256} \pi^{4}+\frac{15 \sqrt{2}}{128} \pi^{4}+\frac{315}{64} \pi^{3}+\frac{45 \sqrt{2}}{32} \pi^{3}+\frac{945}{16} \pi^{2}-\frac{45 \sqrt{2}}{8} \pi^{2}\right) \frac{N}{\omega^{6}} .
\end{aligned}
$$

The coefficients of $N / \omega^{6}$ are approximately equal to 723.0 and 721.4.

The quantities $\beta_{j}$ and $\beta_{j}^{\prime}$ can now be calculated from Eqs. (2.17), when $C_{j}$ is replaced by the expression (2.18) and $B_{j}$ by $(2.21)$. The result is

$$
\beta_{,}=-\frac{1}{15}+\frac{64}{5 \pi^{2}} \frac{2^{2 j}}{2^{2 n}} \frac{1-\left(40 / \pi^{2}\right)\left(2^{2 j} / 2^{2 n}\right)}{1-\left(48 / \pi^{2}\right)\left(2^{2 i} / 2^{2 n}\right)},
$$




$$
\begin{aligned}
\beta_{j}{ }^{\prime} & =\frac{16}{15}-\frac{64}{5 \pi^{2}} \frac{2^{2 j}}{2^{2}} \frac{1-\left(40 / \pi^{2}\right)\left(2^{2 j} / 2^{2 n}\right)}{1-\left(48 / \pi^{2}\right)\left(2^{2 j} / 2^{2 n}\right)}, \quad j=1,2, \cdots, n-2 \\
\beta_{n-1} & =-\frac{1}{15}+\frac{16\left(\pi^{4}+60 \pi^{2}-720\right)}{375 \pi^{2}\left(\pi^{2}-12\right)}, \quad \beta_{n-1}^{\prime}=\frac{16}{15}-\frac{16\left(\pi^{4}+60 \pi^{2}-720\right)}{375 \pi^{2}\left(\pi^{2}-12\right)} \\
\beta_{n} & =-\frac{1}{15}-\frac{32}{15} \frac{\pi^{4}-\pi^{4} \sqrt{2}-42 \pi^{3}-12 \pi^{3} \sqrt{2}-462 \pi^{2}+48 \pi^{2} \sqrt{2}+5760}{(7+2 \sqrt{2}) \pi^{2}\left(\pi^{2}+12 \pi-48\right)} \\
\beta_{n}{ }^{\prime} & =\frac{16}{15}+\frac{32}{15} \frac{\pi^{4}-\pi^{4} \sqrt{2}-42 \pi^{3}-12 \pi^{3} \sqrt{2}-462 \pi^{2}+48 \pi^{2} \sqrt{2}+5760}{(7+2 \sqrt{2}) \pi^{2}\left(\pi^{2}+12 \pi-48\right)}
\end{aligned}
$$

Using these coefficients in Eqs. (2.17), the $C_{j}$ values become exact if $f(x)$ is a polynomial of at most 7 th degree, while in other cases the $C_{j}$-sequence gives results of increasing accuracy.

3. Integration Formulae for $\int_{0}^{N} f(x) \sin \omega x d x$. Approximation of $f(x)$ by a constant yields zero for the integral, but approximation by a linear function leads to

$$
\frac{1}{\omega}\{f(0)-f(N)\} \text {. }
$$

If $f(x)$ is approximated by a different linear function over each half of the interval, the same result appears. For further halving of the interval, this invariance of the result continues until $f(x)$ is approximated over each quarter wavelength $\pi / 2 \omega$ by a different linear function. However, since the results up to this division are invariant, it is neither possible to obtain better results by linear combination. Therefore, the linear approximation is not very suitable to be used as starting point for a scheme comparable to that used for the cosine-integral.

Since the results for linear functions are also exact for 2 nd degree polynomials, the following step could be to derive formulae by approximating $f(x)$ by a $3 \mathrm{rd}$ degree polynomial fitting at the points $x=0, N / 3,2 N / 3$ and $N$.

However, this would lead to points of evaluation for $f(x)$, which differ from those required in the calculation of the cosine-integral. For this reason, it has been preferred to approximate $f(x)$ by a 4th degree polynomial fitting at $x=0, N / 4$, $N / 2,3 N / 4$ and $N$. After some elementary calculations this leads to the first approximation

$$
\begin{aligned}
A_{1}: \frac{1}{\omega}\{f(0)- & \left.f\left(2^{n} \frac{\pi}{\omega}\right)\right\} \\
& -\frac{2^{5-2 n}}{\pi^{2} \omega}\left\{f(0)-2 f\left(2^{n-2} \frac{\pi}{\omega}\right)+2 f\left(3 \cdot 2^{n-2} \frac{\pi}{\omega}\right)-f\left(2^{n} \frac{\pi}{\omega}\right)\right\},
\end{aligned}
$$

where $n$ has been defined by Eq. (2.1).

The second approximation, obtained by aid of two different 4 th degree polynomials, one for each half of the total interval is

$$
\begin{aligned}
A_{2}: \frac{1}{\omega}\left\{f(0)-f\left(2^{n} \frac{\pi}{\omega}\right)\right\} & -\frac{2^{7-2 n}}{\pi^{2} \omega}\left\{f(0)-2 f\left(2^{n-3} \frac{\pi}{\omega}\right)+2 f\left(3 \cdot 2^{n-3} \frac{\pi}{\omega}\right)\right. \\
& \left.-2 f\left(5 \cdot 2^{n-3} \frac{\pi}{\omega}\right)+2 f\left(7 \cdot 2^{n-3} \frac{\pi}{\omega}\right)-f\left(8 \cdot 2^{n-3} \frac{\pi}{\omega}\right)\right\} .
\end{aligned}
$$


Continuing this procedure, we obtain as $j$ th approximation $(j \leqq n)$

$$
\begin{aligned}
A_{j}: \frac{1}{\omega}\{f(0)-f & \left.\left(2^{n} \frac{\pi}{\omega}\right)\right\}-\frac{2^{2 j+3-2 n}}{\pi^{2} \omega}\left[f(0)-f\left(2^{n} \frac{\pi}{\omega}\right)\right. \\
& \left.\left.-2 \sum_{k=1}^{2 j-1}\left[f\{4 k-3) 2^{n-j-1} \frac{\pi}{\omega}\right\}-f\left\{(4 k-1) 2^{n-j-1} \frac{\pi}{\omega}\right\}\right]\right] .
\end{aligned}
$$

The $n$th approximation will be

$$
\begin{aligned}
A_{n}: \frac{1}{\omega}\left(1-\frac{8}{\pi^{2}}\right)\{f(0)- & f(N)\} \\
& +\frac{16}{\pi^{2} \omega} \sum_{k=1}^{p}\left[f\left\{\left(2 k-\frac{3}{2}\right) \frac{\pi}{\omega}\right\}-f\left\{\left(2 k-\frac{1}{2}\right) \frac{\pi}{\omega}\right\}\right],
\end{aligned}
$$

where $p$ is not necessarily equal to $2^{n-1}$. In this last formula $f(x)$ has been approximated by a different 4 th degree polynomial over each wavelength $2 \pi / \omega$.

The next approximation follows by approximating $f(x)$ separately over each half wavelength

$$
\begin{aligned}
A_{n+1}: & \frac{\pi^{2}+2 \pi-16}{\pi^{2} \omega}\{f(0)-f(N)\} \\
+ & \frac{4(3 \pi-8)}{\pi^{2} \omega} \sum_{k=1}^{p}\left[f\left\{\left(2 k-\frac{3}{2}\right) \frac{\pi}{\omega}\right\}-f\left\{\left(2 k-\frac{1}{2}\right) \frac{\pi}{\omega}\right\}\right] \\
& +\frac{8(4-\pi)}{\pi^{2} \omega} \sum_{k=1}^{p}\left[f\left\{\left(2 k-\frac{7}{4}\right) \frac{\pi}{\omega}\right\}+f\left\{\left(2 k-\frac{5}{4}\right) \frac{\pi}{\omega}\right\}\right. \\
& \left.\quad-f\left\{\left(2 k-\frac{3}{4}\right) \frac{\pi}{\omega}\right\}-f\left\{\left(2 k-\frac{1}{4}\right) \frac{\pi}{\omega}\right\}\right] .
\end{aligned}
$$

Finally, the $(n+2)$ th approximation is

$$
\begin{aligned}
& A_{n+2}: \frac{1}{3 \pi^{4} \omega}\left(3 \pi^{4}+6 \pi^{3}-560 \pi^{2}-2304 \pi+12288\right)\{f(0)-f(N)\} \\
& +\frac{4}{3 \pi^{4} \omega}\left(25 \pi^{3}-88 \pi^{2}-1920 \pi+6144\right) \sum_{k=1}^{p}\left[f\left\{\left(2 k-\frac{3}{2}\right) \frac{\pi}{\omega}\right\}\right. \\
& +\frac{8}{\pi^{4} \omega}\left(3 \pi^{3}-76 \pi^{2}-768 \pi+3072\right) \sum_{k=1}^{p}\left[f\left\{\left(2 k-\frac{7}{4}\right) \frac{\pi}{\omega}\right\}\right. \\
& \left.+f\left\{\left(2 k-\frac{5}{4}\right) \frac{\pi}{\omega}\right\}-f\left\{\left(2 k-\frac{3}{4}\right) \frac{\pi}{\omega}\right\}-f\left\{\left(2 k-\frac{1}{4}\right) \frac{\pi}{\omega}\right\}\right] \\
& +\frac{32}{3 \pi^{4} \omega}\left(-\pi^{3}+52 \pi^{2}+336 \pi-1536\right) \sum_{k=1}^{p}\left[f\left\{\left(2 k-\frac{15}{8}\right) \frac{\pi}{\omega}\right\}\right. \\
& \left.+f\left\{\left(2 k-\frac{9}{8}\right) \frac{\pi}{\omega}\right\}-f\left\{\left(2 k-\frac{7}{8}\right) \frac{\pi}{\omega}\right\}-f\left\{\left(2 k-\frac{1}{8}\right) \frac{\pi}{\omega}\right\}\right] \\
& +\frac{32}{3 \pi^{4} \omega}\left(-3 \pi^{3}+28 \pi^{2}+432 \pi-1536\right) \sum_{k=1}^{p}\left[f\left\{\left(2 k-\frac{13}{8}\right) \frac{\pi}{\omega}\right\}\right. \\
& \left.+f\left\{\left(2 k-\frac{11}{8}\right) \frac{\pi}{\omega}\right\}-f\left\{\left(2 k-\frac{5}{8}\right) \frac{\pi}{\omega}\right\}-f\left\{\left(2 k-\frac{3}{8}\right) \frac{\pi}{\omega}\right\}\right] .
\end{aligned}
$$


The formulae (3.4), (3.5) and (3.6) have again the favourable property that for any value of $x$ the contribution of $f(x)$ has the same sign as $f(x)$ sin $\omega x$. These same formulae can also be used to calculate arbitrary Fourier sine coefficients of $f(x)$ in the interval $(0, N)$.

All formulae of the $A_{j}$-sequence in this chapter are exact if $f(x)$ is a polynomial of at most 4 th degree.

By linear combination of two consecutive results of the $A_{j}$-sequence it is possible to obtain formulae which are exact for polynomials of 5 th degree and, by reasons of symmetry, which are then also exact for polynomials of 6 th degree (over intervals of $2^{n-j}$ wavelengths). The exact result for $f(x)=x^{5}$ is given by

$$
\int_{0}^{N} x^{5} \sin \omega x d x=-\frac{N^{5}}{\omega}+20 \frac{N^{3}}{\omega^{3}}-120 \frac{N}{\omega^{5}} .
$$

Approximate results are

$$
\begin{aligned}
A_{j}: & -\frac{N^{5}}{\omega}+\left(20-\frac{25}{2^{2 j+1}}\right) \frac{N^{3}}{\omega^{3}}, \quad j=1,2, \cdots, n, \\
A_{n+1}: & -\frac{N^{5}}{\omega}+20 \frac{N^{3}}{\omega^{3}}-\left(\frac{85}{8} \pi^{2}+\frac{15}{32} \pi^{3}\right) \frac{N}{\omega^{5}}, \\
A_{n+2}: & -\frac{N^{5}}{\omega}+20 \frac{N^{3}}{\omega^{3}}-120 \frac{N}{\omega^{5}} .
\end{aligned}
$$

The last expression shows that $A_{n+2}$ is already exact for polynomials of at most 6 th degree.

The coefficients $\alpha_{j}$ and $\alpha_{j}^{\prime}$ are determined by Eqs. (2.9) and (2.10), substituting for $B_{j}$ the exact value (3.7). Then

$$
\begin{aligned}
\alpha_{j} & =-\frac{1}{3}+\frac{64}{5 \pi^{2}} \cdot \frac{2^{2 j}}{2^{2 n}}, \quad \alpha_{j}{ }^{\prime}=\frac{4}{3}-\frac{64}{5 \pi^{2}} \cdot \frac{2^{2 j}}{2^{2 n}}, \quad j=1,2, \cdots, n-1, \\
\alpha_{n} & =-\frac{1}{3}+\frac{4\left(\pi^{3}+16 \pi^{2}-192\right)}{3 \pi^{2}(\pi-4)}, \quad \alpha_{n}{ }^{\prime}=\frac{4}{3}-\frac{4\left(\pi^{3}+16 \pi^{2}-192\right)}{3 \pi^{2}(\pi-4)}, \\
\alpha_{n+1} & =0, \quad \alpha_{n+1}^{\prime}=1 .
\end{aligned}
$$

If $f(x)$ differs from a 6 th degree polynomial, the $B_{j}$-sequence formed with the coefficients (3.8) gives results of increasing accuracy.

By linear combination of two consecutive results of the $B_{j}$-sequence, it is again possible to obtain results which are exact for polynomials up to the 8th degree. The exact result for $f(x)=x^{7}$ is given by

$$
\int_{0}^{N} x^{7} \sin \omega x d x=-N_{\omega}^{7}+42 \frac{N^{5}}{\omega^{3}}-840 \frac{N^{3}}{\omega^{5}}+5040 \frac{N}{\omega^{7}} .
$$

Approximate results are

$$
A_{j}: \quad-\frac{N^{7}}{\omega}+\left(42-\frac{175}{2^{2 j+1}}+\frac{427}{2^{4 j+3}}\right) \frac{N^{5}}{\omega^{3}}, \quad j=1,2, \cdots, n,
$$


$A_{n+1}:-\frac{N^{7}}{\omega}+42 \frac{N^{5}}{\omega^{3}}-\left(\frac{105}{32} \pi^{3}+\frac{595}{8} \pi^{2}\right) \frac{N^{3}}{\omega^{5}}+\left(\frac{1155}{512} \pi^{5}-\frac{5677}{128} \pi^{4}\right) \frac{N}{\omega^{7}}$,

$A_{n+2}: \quad-\frac{N^{7}}{\omega}+42 \frac{N^{5}}{\omega^{3}}-840 \frac{N^{3}}{\omega^{5}}+\left(-\frac{105}{2048} \pi^{5}-\frac{1267}{512} \pi^{4}+\frac{1575}{64} \pi^{3}+\frac{3675}{8} \pi^{2}\right) \frac{N}{\omega^{7}}$.

Besides the series given by Eqs. (2.13) and (2.20), we used also

$$
\begin{aligned}
& \sum_{k=1}^{m} k^{5}=\frac{1}{6} m^{6}+\frac{1}{2} m^{5}+\frac{5}{12} m^{4}-\frac{1}{12} m^{2}, \\
& \sum_{k=1}^{m} k^{6}=\frac{1}{7} m^{7}+\frac{1}{2} m^{5}-\frac{1}{6} m^{3}+\frac{1}{42} m .
\end{aligned}
$$

Establishing the $B_{j}$-approximations by aid of the coefficients $\alpha_{j}$ and $\alpha_{j}^{\prime}$ given in (3.8), we find

$$
\begin{aligned}
B_{j}: & -\frac{N^{7}}{\omega}+\left(42-\frac{427}{2^{4 j+5}}\right) \frac{N^{5}}{\omega^{3}}-\left(840-\frac{1281}{2^{2 j+1}}\right) \frac{N^{3}}{\omega^{5}}, \quad j=1,2, \cdots, n-1, \\
B_{n}: & -\frac{N^{7}}{\omega}+42 \frac{N^{5}}{\omega^{3}}-840 \frac{N^{3}}{\omega^{5}}+\left(-\frac{217}{32} \pi^{4}+\frac{1155}{2} \pi^{2}\right) \frac{N}{\omega^{7}}, \\
B_{n+1}: & -\frac{N^{7}}{\omega}+42 \frac{N^{5}}{\omega^{3}}-840 \frac{N^{3}}{\omega^{5}}+\left(-\frac{105}{2048} \pi^{5}-\frac{1267}{512} \pi^{4}+\frac{1575}{64} \pi^{3}+\frac{3675}{8} \pi^{2}\right) \frac{N}{\omega^{7}} .
\end{aligned}
$$

Linear combination of two consecutive $B_{j}$-results in the way of Eqs. (2.17) yields exact values for $C_{j}$ if $f(x)$ is a polynomial up to the 8 th degree. We have to take

$$
\begin{aligned}
\beta_{j} & =-\frac{1}{15}+\frac{64}{25 \pi^{2}} \frac{2^{2 j}}{2^{2 n}} \frac{1-\left(600 / 61 \pi^{2}\right)\left(2^{2 j} / 2^{2 n}\right)}{1-\left(48 / 5 \pi^{2}\right)\left(2^{2 j} / 2^{2 n}\right)}, \\
\beta_{j}^{\prime} & =\frac{16}{15}-\frac{64}{25 \pi^{2}} \frac{2^{2 j}}{2^{2 n}} \frac{1-\left(600 / 61 \pi^{2}\right)\left(2^{2 j} / 2^{2 n}\right)}{1-\left(48 / 5 \pi^{2}\right)\left(2^{2 j} / 2^{2 n}\right)}, \quad j=1,2, \cdots, n-2, \\
\beta_{n-1} & =-\frac{217 \pi^{4}-18480 \pi^{2}+161280}{6615 \pi^{4}-63504 \pi^{2}}, \quad \beta_{n-1}^{\prime}=\frac{6832 \pi^{4}-81984 \pi^{2}+161280}{6615 \pi^{4}-63504 \pi^{2}} \\
\beta_{n} & =\frac{\left.(15 / 2048) \pi^{5}+(181 / 512) \pi^{4}-(225 / 64) \pi^{3}-525 / 8\right) \pi^{2}+720}{(15 / 2048) \pi^{5}-(315 / 512) \pi^{4}-(225 / 64) \pi^{3}+(135 / 8) \pi^{2}} \\
\beta_{n}{ }^{\prime} & =\frac{-(31 / 32) \pi^{4}+(165 / 2) \pi^{2}-720}{(15 / 2048) \pi^{5}-(315 / 512) \pi^{4}-(225 / 64) \pi^{2}+(135 / 8) \pi^{2}} .
\end{aligned}
$$

For arbitrary $f(x)$ the $C_{j}$-sequence gives approximations of increasing accuracy.

4. Some Remarks. Both for the cosine- and for the sine-integral, the following scheme of approximations is obtained

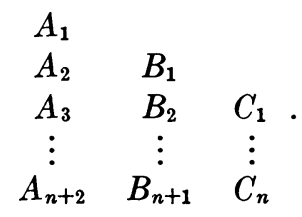


The results are obtained line after line. A tolerance can be built in in the programme, making that the calculations are stopped as soon as, for instance, three consecutive $C_{j}$-values differ by an amount smaller than the tolerance. If not yet $3 C_{j}$-values are available, the tolerance test can be performed on $A_{3}, B_{2}, C_{1}$ or on $B_{3}, C_{1}$ and $C_{2}$. In principle, the scheme could be extended both downward and to the right, but this would require further derivations of formulae. For large values of $\omega$ this will, in general, not be necessary. Then the approximation of $f(x)$ by a polynomial of 7 th or 8 th degree over each wavelength $2 \pi / \omega$ will be sufficiently accurate. The fact that the method works better if $\omega$ is larger makes that it is in some sense complementary to the usual Stiefel procedure.

If the tolerance is not satisfied, the value $C_{n}$ will, in general, be the best approximation. In Section 5 an estimation for the remainder term is given.

In many cases it may be preferable to divide the whole interval $(0, N)$ in subintervals. In the first place this has the advantage that if somewhere in the interval $f(x)$ is difficult to approximate by polynomials, which leads to many evaluation points there, it is not necessary to take the evaluation points in the whole interval at such a small distance. In the second place, if $p$ is some arbitrary integer, it can be written as a sum of powers of two. For those subintervals which contain a number of wavelengths, which is equal to 2 or some higher power of two, the possibility exists that the tolerance is satisfied before $f(x)$ has to be evaluated at 16 points per wavelength.

A number of constants appeared in the integration formulae. They are listed below together with their numerical values.

$\frac{2}{\pi}$

$$
\begin{aligned}
& =+0.636619772368 \\
& =+0.288720378825 \\
& =+0.695798787084 \\
& =+0.134761604357 \\
& =+0.190581688568 \\
& =+0.476323248443 \\
& =+0.197299549579 \\
& =+0.101321183642 \\
& =-0.053308755960 \\
& =-0.063484367498
\end{aligned}
$$$$
\frac{2}{\pi}\left(3-\frac{8}{\pi}\right)
$$$$
\frac{8}{\pi}\left(\frac{4}{\pi}-1\right)
$$$$
\frac{2}{\pi}\left(6+\sqrt{ } 2-\frac{16 \sqrt{ } 2}{\pi}\right)
$$$$
\frac{4}{\pi}\left(1+3 \sqrt{ } 2-\frac{16}{\pi}\right)
$$$$
\frac{8}{\pi}\left(\frac{8 \sqrt{ } 2}{\pi}-2-\sqrt{ } 2\right)
$$$$
\frac{8}{\pi}\left(\frac{16-8 \sqrt{ } 2}{\pi}-\sqrt{ } 2\right)
$$$$
\frac{1}{\pi^{2}}
$$$$
-\frac{1}{3}+\frac{16(\pi-3)}{3 \pi(4-\pi)}
$$$$
-\frac{1}{3}+\frac{16(\pi+2 \pi \sqrt{ } 2-12)}{3 \pi(8 \sqrt{ } 2-\pi-8)}
$$ 


$$
\begin{aligned}
& -\frac{1}{15}+\frac{16\left(\pi^{4}+60 \pi^{2}-720\right)}{375 \pi^{2}\left(\pi^{2}-12\right)} \\
& =-0.004948720738 \\
& -\frac{1}{15}+\frac{32}{15} \cdot \frac{\pi^{4}-\pi^{4} \sqrt{2}-42 \pi^{3}-12 \pi^{3} \sqrt{2}-462 \pi^{2}+48 \pi^{2} \sqrt{2}+5760}{(7+2 \sqrt{ } 2) \pi^{2}\left(\pi^{2}+12 \pi-48\right)} \\
& =+0.005130603384 \\
& 1-\frac{8}{\pi^{2}} \\
& =+0.189430530861 \\
& \frac{\pi^{2}+2 \pi-16}{\pi^{2}} \\
& =+0.015480834090 \\
& \frac{1}{3 \pi^{4}}\left(3 \pi^{4}+6 \pi^{3}-560 \pi^{2}-2304 \pi+12288\right) \\
& =+0.003617029621 \\
& \frac{4}{3 \pi^{4}}\left(25 \pi^{3}-88 \pi^{2}-1920 \pi+6144\right) \\
& =+0.256975806809 \\
& \frac{8}{\pi^{4}}\left(3 \pi^{3}-76 \pi^{2}-768 \pi+3072\right) \\
& =+0.179509947414 \\
& \frac{32}{3 \pi^{4}}\left(-\pi^{3}+52 \pi^{2}+336 \pi-1536\right) \\
& =+0.195823888828 \\
& \frac{32}{3 \pi^{4}}\left(-3 \pi^{3}+28 \pi^{2}+432 \pi-1536\right) \\
& =+0.492561230732 \\
& -\frac{1}{3}+\frac{4\left(\pi^{3}+16 \pi^{2}-192\right)}{3 \pi^{2}(\pi-4)} \\
& =+0.151400955182 \\
& -\frac{217 \pi^{4}-18480 \pi^{2}+161280}{6615 \pi^{4}-63504 \pi^{2}} \\
& =-0.001561400230 \\
& \frac{(15 / 2048) \pi^{5}+(181 / 512) \pi^{4}-(225 / 64) \pi^{3}+(525 / 8) \pi^{2}+720}{(15 / 2048) \pi^{5}-(315 / 512) \pi^{4}-(225 / 64) \pi^{3}+(135 / 8) \pi^{2}} \\
& =+0.153446190363
\end{aligned}
$$

The texts of procedures written in ALGOL 60 is available by request to the authors.

5. The Remainder Term. The error in the integration formulae can be investigated by aid of the theory given by Milne [8, $\$ 30$ and 31]. The error over one wavelength is defined as

$$
R(f)=\int_{0}^{a} f(x) \underset{\sin }{\cos } \omega x d x-\text { value given by integration formula, }
$$

where $R(f)$ is a linear operation to be performed on the function $f(x)$ and $a=2 \pi / \omega$. We shall say that the operator $R$ is of degree $n$ when

$$
R\left(x^{m}\right)=0 \quad \text { if } m \leqq n \text { and } R\left(x^{n+1}\right) \neq 0 .
$$

The operator giving the error of the result $C_{n}$ is of degree 7 in the case of the cosine-integral and of degree 8 in the case of the sine-integral. 
It is shown by Milne that for an operator of degree $n$, one has

$$
R(f)=\int_{-\infty}^{\infty} f^{(n+1)}(s) G(s) d s
$$

where $G(s)$ is the result of applying the operator $R$ to the function $(\overline{x-s})^{n} / n$ ! regarded as a function of the variable $x$. The function $(\overline{x-s})^{n}$ is defined by

$$
\begin{array}{ll}
(\overline{x-s})^{n}=(x-s)^{n} & \text { if } x>s, \\
(\overline{x-s})^{n}=0 & \text { if } x<s .
\end{array}
$$

If $G(s)$, which only depends on the operator $R$ but not on the function $f$, does not change sign, the operator $R$ is definite and then the equation for $R(f)$ becomes

$$
R(f)=f^{(n+1)}(\xi) R\left\{\frac{x^{n+1}}{(n+1) !}\right\}
$$

where $\xi$ is some value of $x$ in the range of integration.

The practical difficulty is that (5.2) is much easier to evaluate than (5.1), but that on the other hand (5.2) only holds for definite operators $R$ and that for showing $R$ to be definite it is necessary to show that $G(s)$ does not change sign. This has been shown by the authors [9] for the operator corresponding to $C_{n}$ in the case of the cosine-integral and it can be shown for the sine-integral along similar lines. Hence Eq. (5.2) is valid for estimating the error. Thus we have to calculate $R\left\{x^{8} / 8\right.$ ! $\}$ for obtaining the error in the formula for the cosine-integral.

The exact value of

$$
\int_{0}^{a} f(x) \cos \omega x d x \quad \text { with } f(x)=\frac{x^{8}}{8 !} \quad \text { and } \quad \omega a=2 \pi
$$

is equal to

$$
\frac{30.159127404}{\omega^{9}}
$$

The approximation $C_{n}$, calculated by the program, gives for this case

$$
\frac{30.159221885}{\omega^{9}} \text {. }
$$

Hence, it can be concluded that the remainder term for the approximation $C_{n}$ over one wavelength $a$ is given by

$$
-\frac{9.4481 * 10^{-5}}{\omega^{9}} f^{(8)}(\xi) .
$$

If the whole interval of integration, length $N$, corresponds to $p$ wavelengths, the remainder term becomes

$$
-1.5 * 10^{-5} * \frac{N}{\omega^{8}} f^{(8)}(\xi)
$$

where $\xi$ lies somewhere in the interval. 
Similar derivations hold for the sine-integral with the result that then the remainder term for the $C_{n}$-approximation over a length $N$ becomes

$$
+1.1 * 10^{-7} \frac{N}{\omega^{9}} f^{(9)}(\xi)
$$

where $\xi$ is somewhere in the interval.

Mathematical Institute

University of Groningen

Reitdiepskade 4

Groningen, The Netherlands

1. E. STIEFEL, Einführung in die numerische Mathematik, Teubner, Stuttgart, 1961, pp. 131-137. MR 22 \#12686.

2. L. Fox, Numerical Solution of Ordinary and Partial Differential E'quations, Pergamon, Oxford, 1962. MR 26 \#4488.

3. L. N. G. FiLON, "On a quadrature formula for trigonometric integrals," Proc. Roy. Soc. Edinburgh Sect. A, v. 49, 1929, pp. 38-47.

4. Y. L. LUke, "On the computation of oscillatory integrals," Proc. Cambridge Philos. Soc., v. 50, 1954, pp. 269-277. MR 15, 992.

5. H. HURWITZ \& P. F. ZWEIFEL, "Numerical quadrature of Fourier transform integrals," $M T A C$, v. 10,1956 , pp. 142-149. MR 18, 337.

6. H. HuRwitz, R. A. PFeiffer \& P. F. Zweifel, "Numerical quadrature of Fourier transform integrals. II," MTAC, v. 13, 1959, pp. 87-90. MR 21 \#5283.

7. A. SÄNGER, "On numerical quadrature of Fourier transforms," J. Math. Anal. Appl., v. 8,1964 , pp. 1-3. MR $28 \# 720$.

8. W. E. Milne, Numerical Calculus, Princeton Univ. Press, Princeton, N. J., 1949. MR 10,483

9. A. I. VAN DE VOOREN \& H. J. VAN LINDE, "Numerical calculation of integrals with strongly oscillating integrand," Report TW-22, Math. Inst., University of Groningen, 1965. 